\title{
PLANS AND OBJECTIVES OF THE REMAINING APOLLO MISSIONS
}

\author{
L. R. SCHERER \\ Director, Apollo Lunar Exploration, Apollo Program Office, National Aeronautics \\ and Space Administration, Washington, D.C., U.S.A.
}

\begin{abstract}
The three remaining Apollo missions will have significantly increased scientific capabilities. These result from increased payload, more time on the surface, improved range, and more sophisticated experiments on the surface and in orbit. Landing sites for the last three missions will be carefully selected to maximize the total scientific return.
\end{abstract}

There are three remaining Apollo missions. For these missions we have been able to incorporate major increases in system capabilities and experimental payloads over that of the earlier missions. This is summarized in Figure 1. The landed payload capability will be greater than $1200 \mathrm{lbs}$. The astronauts will be able to make three

- Landed scientific payload doubled.

- Addition of service module experiment package.

- Lunar surface stay time doubled.

- Eva manhours increased from 18 to 40 .

- Increased range and efficiency of surface operations.

- Improved suit mobility.

Improved life support system.

- Lunar roving vehicle.

Fig. 1. Increased capabilities Apollo 15 mission.

separate exursions on the surface, each of greater duration than in the past. Major new experiments are planned, particularly for use in orbit. The operational capabilities of the crew have been substantially increased by improved life support systems, suits and mobility.

Figure 2 is a list of the surface experiment payloads as they are now planned. The list is too long to discuss each experiment individually, but let me invite attention to just a couple. Note the traverse type experiments planned for use with the Rover for Apollo 17. These are the surface electrical properties, the traverse gravimeter and the lunar seismic profiling. It is hoped that these complementary experiments will contribute much to our understanding of the near sub-surface of the Moon. On Apollo 17 we do not plan to carry a passive seismometer. In its place we are developing a tidal gravimeter whose data may prove to be of particular significance. The primary function of this instrument is to measure the tidal acceleration of the lunar surface. However, it is so sensitive that it may be able to detect gravitational waves originally predicted by Einstein, if such things actually exist. Experiments searching for such proof conducted on Earth are generally considered controversial. Using the Moon 


\begin{tabular}{llllll}
\hline & Experiment & Investigator & 15 & 16 & 17 \\
\hline S-031 & Passive seismic & Latham & $\times$ & $\times$ & $(\times)$ \\
S-033 & Active seismic & Kovach & & $\times$ & \\
S-034 & Lunar surface magnetometer & Sonett/Dyal & $\times$ & $\times$ & \\
S-035 & Solar wind spectrometer & Snyder & $\times$ & & \\
S-036 & Suprathermal ion detector & Freeman & $\times$ & & \\
S-037 & Heat flow & Langseth & $\times$ & $\times$ & $\times$ \\
S-038 & Charged particle lunar environment & O'brien/Reasoner & & & \\
S-058 & Cold cathode ionization & Johnson & $\times$ & & \\
M-515 & Lunar dust detector & Freden & $\times$ & & \\
S-207 & Lunar surface gravimeter & Weber & & & $(\times)$ \\
S-202 & Lunar ejects and meteorites & Berg & & & $\times$ \\
S-203 & Lunar seismic profiling & Kovach & & & $\times$ \\
S-205 & Lunar atmosphereic composition & Hoffman & & & $\times$ \\
S-201 & Far UV camera/spectroscope & Carruthers & & $\times$ & \\
S-059 & Lunar geology investigation & Swann/Muelberger & $\times$ & $\times$ & $\times$ \\
S-078 & Laser ranging retro-reflector & Faller & $\times$ & & \\
S-080 & Solar wind composition & Geiss & $\times$ & & \\
& Lunar surface close-up camera & Facility & & & \\
S-152 & Cosmic ray detector & Fleischer/Walker/Price & & $\times$ & \\
S-198 & Lunar portable magnetometer & Dyal & & $\times$ & \\
S-199 & Lunar gravity traverse & Talwani & & & $\times$ \\
S-200 & Soil mechanics & Mitchell & $\times$ & $\times$ & $\times$ \\
S-204 & Surface electrical properties & Simmons & & & $\times$ \\
\hline
\end{tabular}

Fig. 2. Apollo lunar surface science plan.

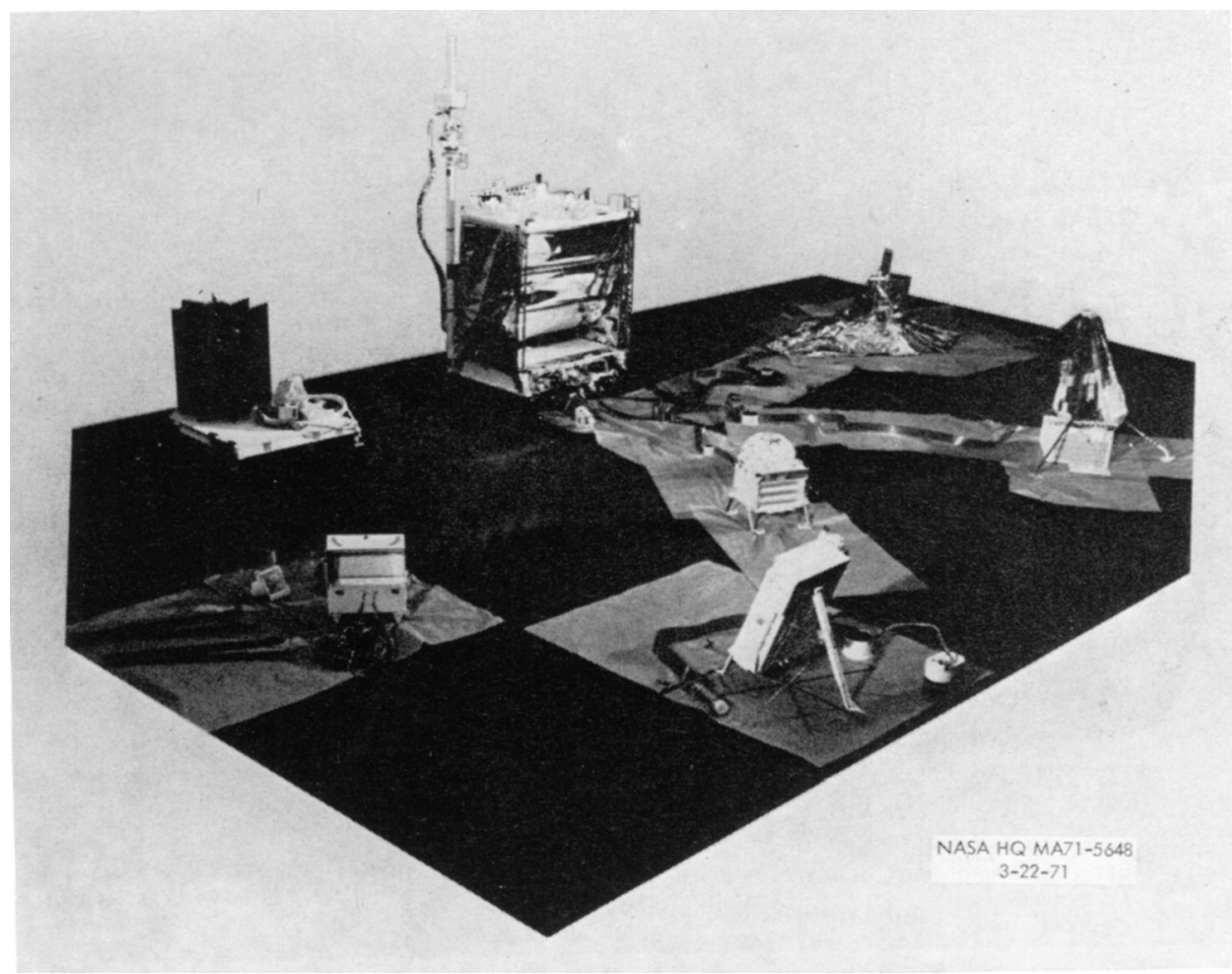

Fig. 3. Apollo 15 geophysical station. 


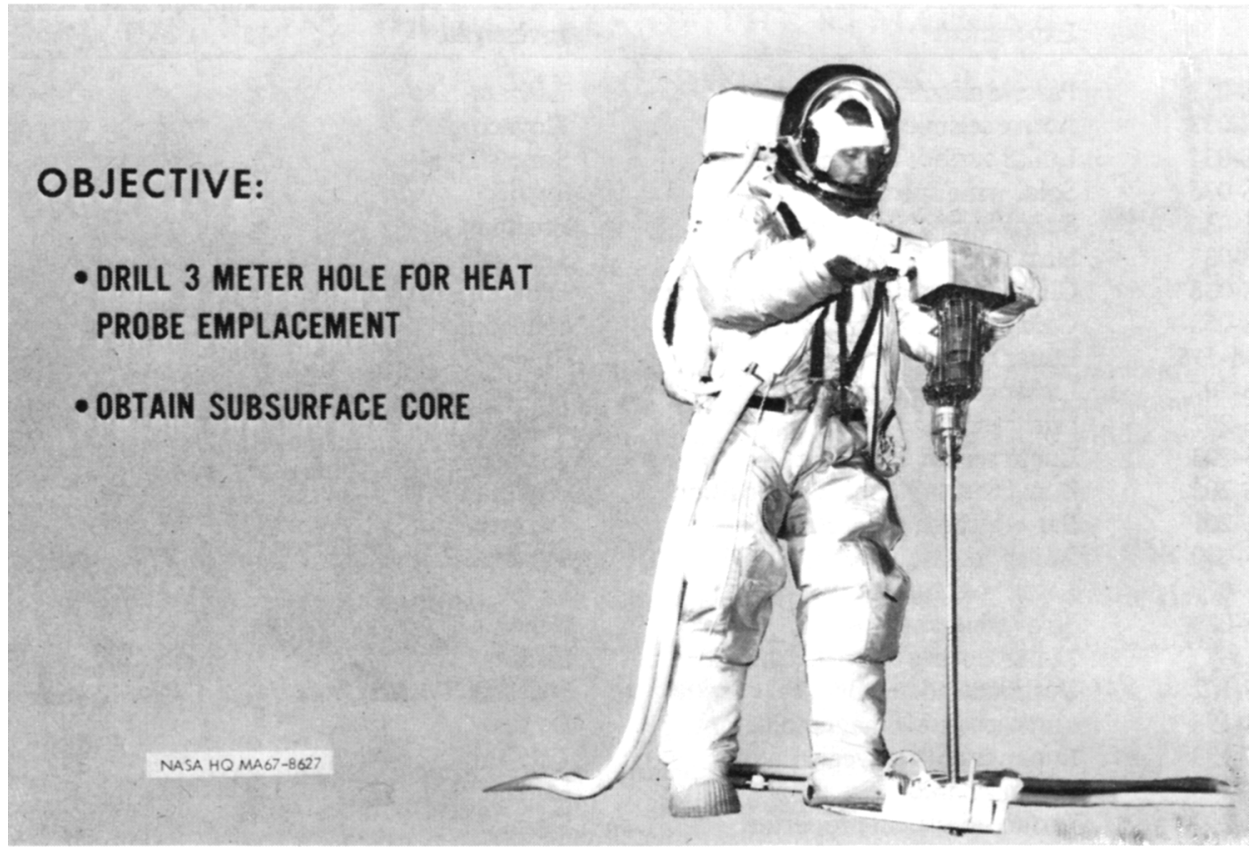

Fig. 4. Lunar surface drill.

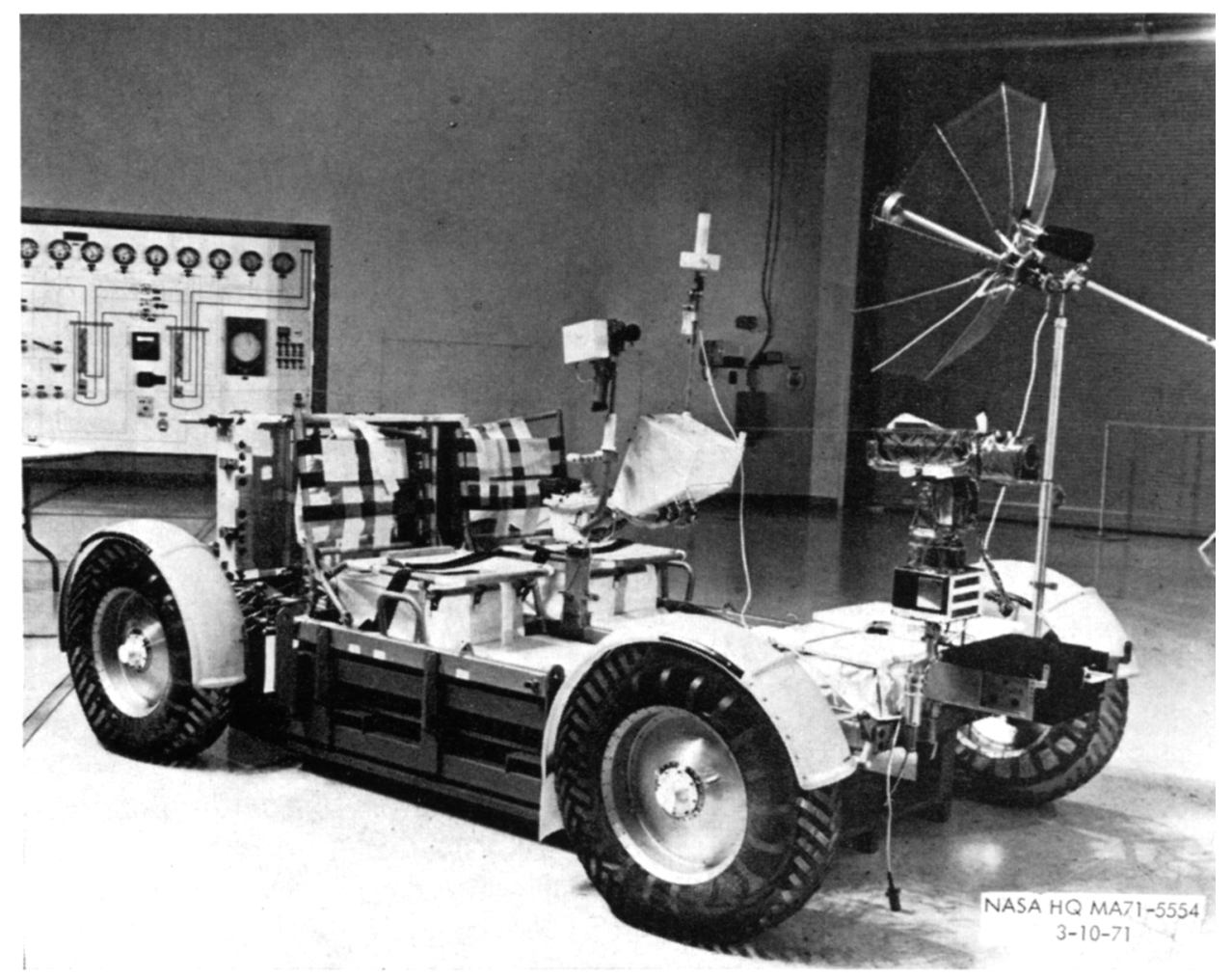

Fig 5. Rover. 
Experiment

Service module:

S-170

S-160

S-161

S-162

S-165

S-173

S-174

S-164

S-169

S-171

S-167/S-168

S-164

S-176

S-177

S-178

Bistatic radar

Laser altimeter

Subsatellite:

Lunar sounder
Gamma-ray spectrometer

$\mathrm{X}$-ray fluorescence

Alpha-particle spectrometer

Mass spectrometer

SM Photographic tasks: (Photo Team)

24" panoramic camera

3" mapping camera

Particle shadows/boundary layer

Subsatellite magnetometer

S-band transponder

Far UV spectrometer

IR Scanning radiometer

S-band transponder (CSM/LM)

Command module:

CM Photographic tasks: (Photo Team)

Apollo window meteoroid

UV Photo-Earth and Moon

Gegenschein from lunar orbit
Investigator

$\begin{array}{lll}15 & 16 & 17\end{array}$

Howard

Arnold

Adler

Gorenstein

Hoffman

(None)

$\begin{array}{lll}\text { Anderson } & \times & \times \\ \text { Coleman } & \times & \times \\ \text { Sjogren } & \times & \times\end{array}$

Fastie

Low

Ward/Brown

Sjogren

(None)

Flaherty

Owen

Dunkelman

Fig. 6. Apollo lunar orbital experiments and photographic tasks.

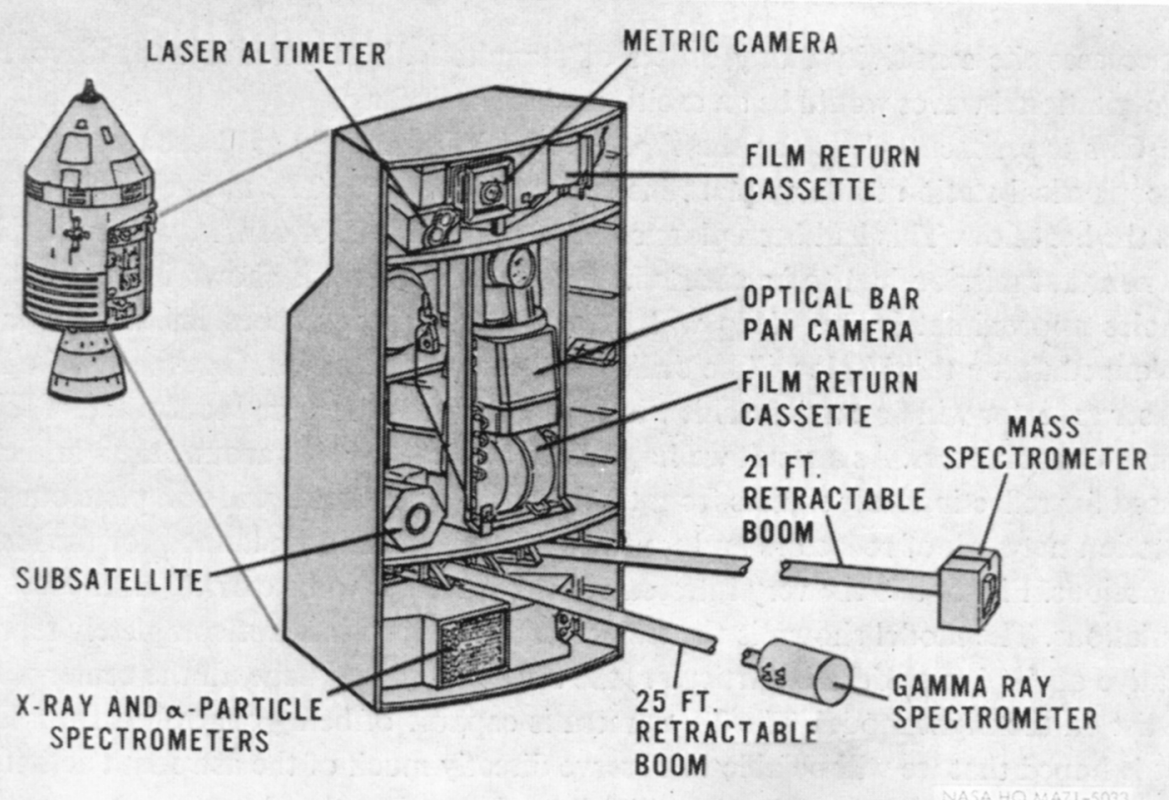

Fig. 7 Apollo orbital science instrument module. 


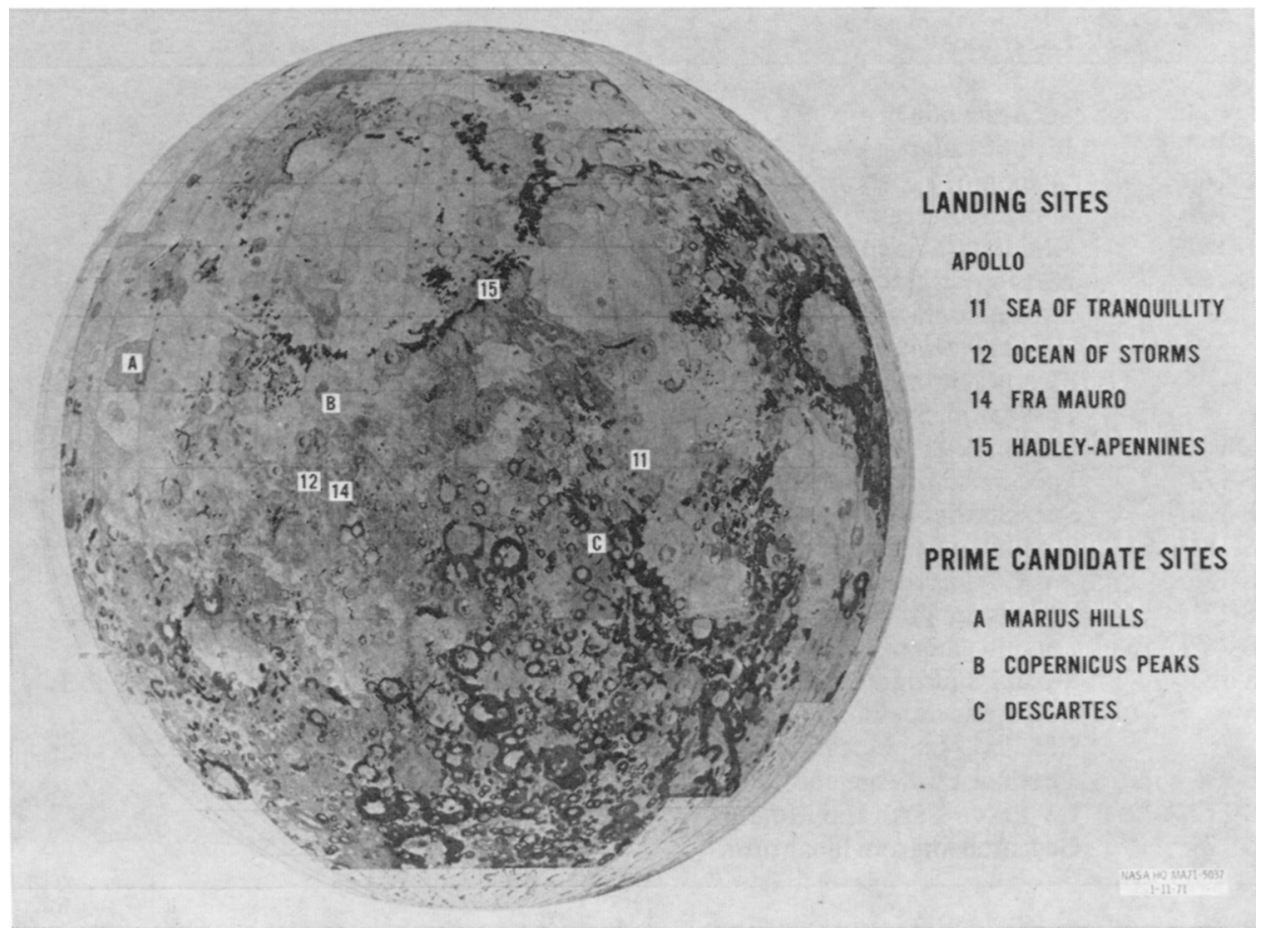

Fig. 8. Geologic map of the Moon with landing sites.

increases the sensitivity many orders of magnitude. The unambiguous discovery of gravitational waves would be an exciting scientific finding.

Our experiment plans for the Apollo 15 mission in July 1971 are quite firm. The geophysical station to be emplaced is shown in Figure 3. The major new experiment is the heat flow. This had been planned for Apollo 13 but, of course, was not deployed when that mission had to be aborted. The drill to be used is shown in Figure 4. Two holes approximately $3 \mathrm{~m}$ deep will be drilled and heat probes inserted. It is also planned that a third hole will be drilled and the core returned. On Apollo 15 a larger laser reflector will be carried. This will have 300 cubes compared to the 100 of the two previous reflectors. As a result we hope that smaller telescopes around the world can be used for reflecting laser signals from the Moon. This reflector will complete our threestation network of reflectors and no further instruments are planned for the last two missions. Figure 5 is the very interesting roving vehicle to be carried on the last three missions. The model shown is the trainer used at $1 \mathrm{~g}$ so it is not completely representative of the $\frac{1}{6} \mathrm{~g}$ model. In particular, the structure shown beneath the center will not exist on the flight model. The TV camera is capable of being controlled from Earth. It is hoped that we will be able to observe directly much of the astronaut activities in their very long traverses. The TV will follow them when they leave the Rover at each station. When the surface mission is completed the Rover will be placed in such a 
position that the actual lift-off from the surface may be observed by the television camera.

As a potential added bonus, by coincidence a lunar eclipse will occur several days after the astronauts leave the Moon. It is hoped that sufficient battery power will remain so the color television can be used to observe the changes in appearance of the lunar surface during the progress of this eclipse.

Figure 6 represents the extensive orbital experiments planned for the final three missions. Excellent panoramic and mapping cameras will be carried and operated in conjunction with the laser altimeter. A set of geochemical sensors is planned for Apollo 15 and 16. These may prove to be very significant for extrapolating the data obtained from specific landing sites over wide regions of the lunar surface. Also on Apollo 15 and 16 we plan to carry small subsatellites. They will remain operative for many months after the manned portion of the mission is completed, providing magnetic and particle fields data. They also should provide greatly improved information on mass concentrations first noted from Lunar Orbiter data. A sophisticated experiment we have under development for the final mission is an electromagnetic sounder which will probe far beneath the lunar surface. Figure 7 is a sketch of the orbital

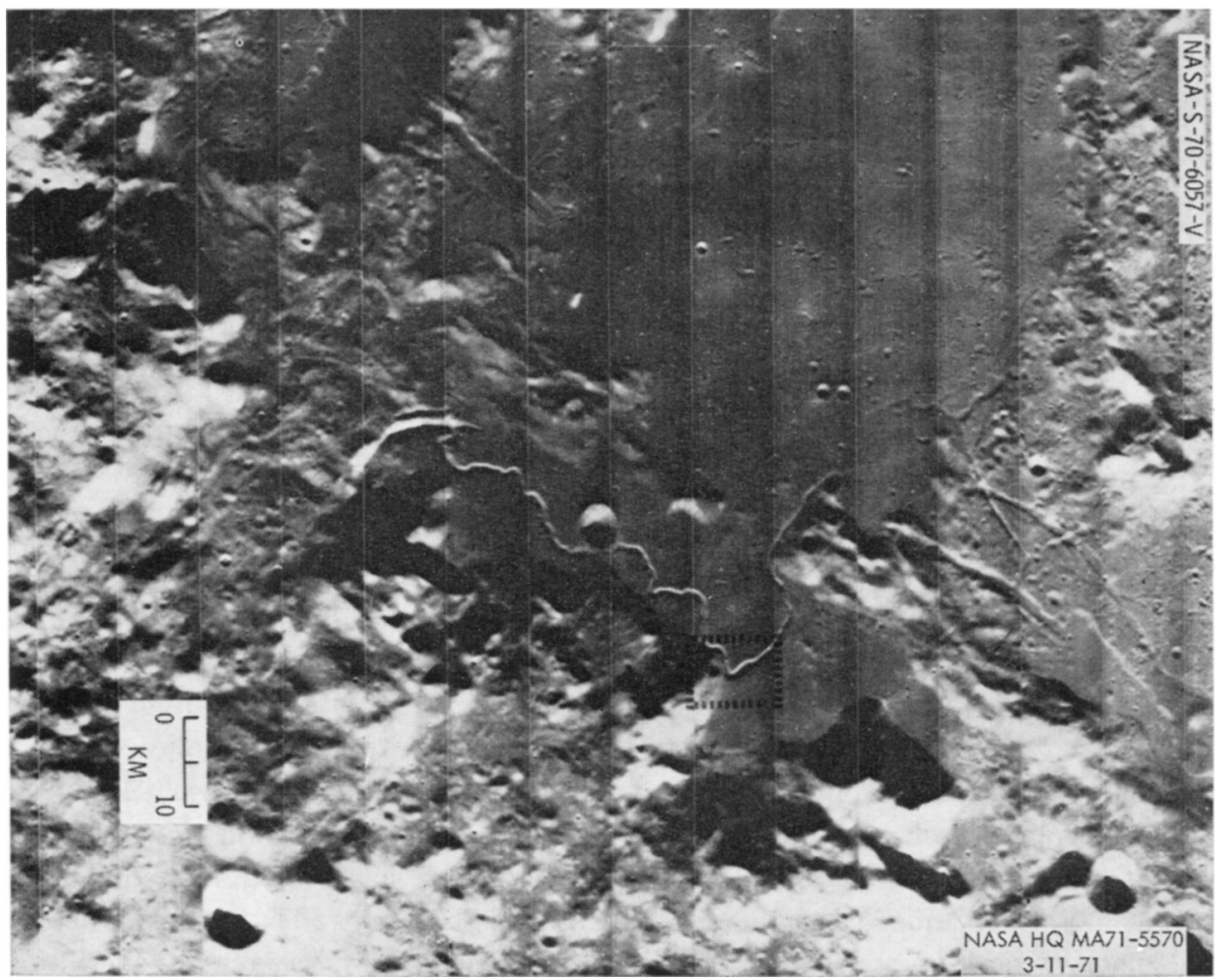

Fig. 9. Hadley Rille. 


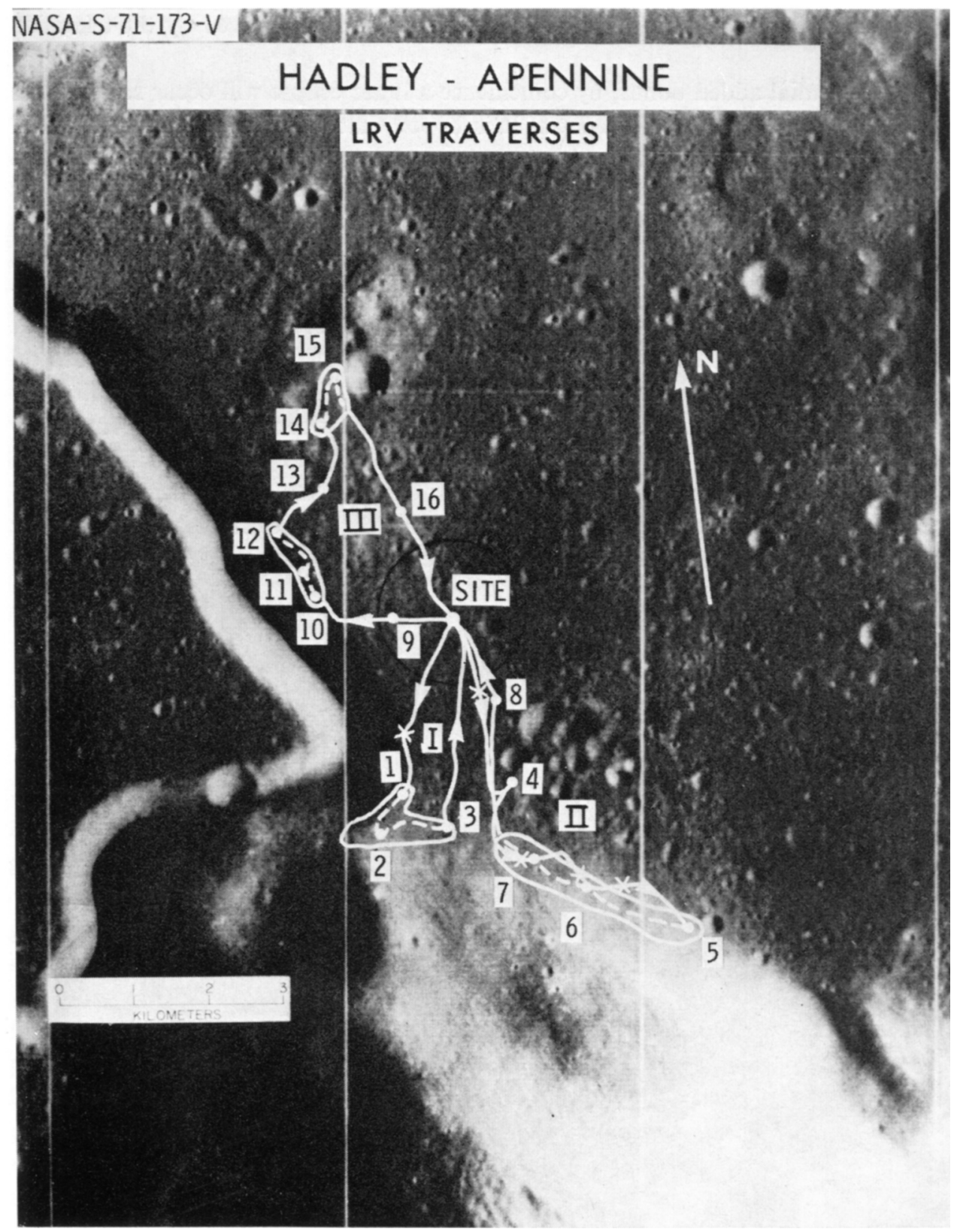

Fig. 10. Close-up view of Hadley-Apennine traverses. 


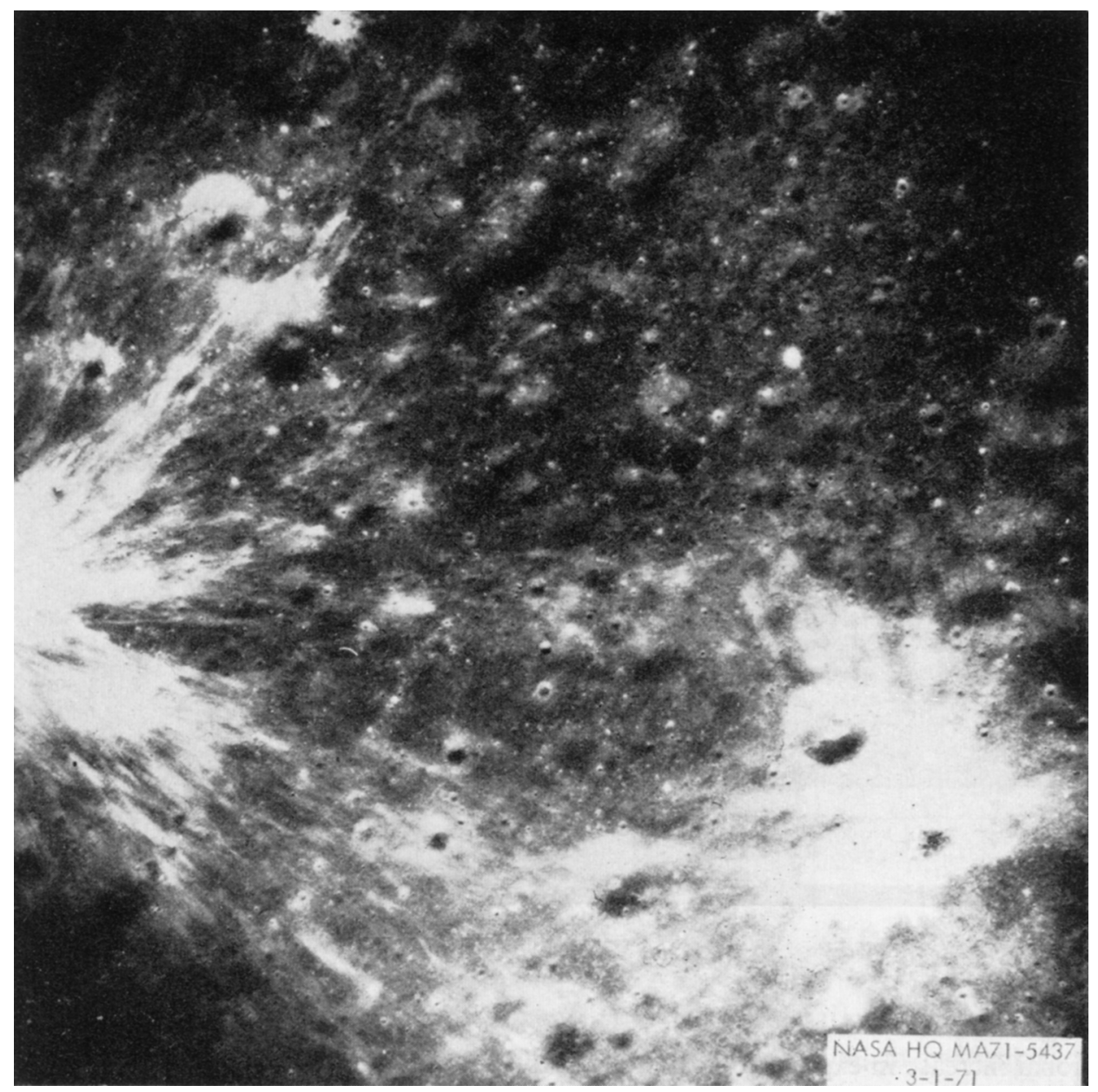

Fig. 11. Descartes.

experiment payload as it is planned for incorporation in the Service Module of Apollo 15. With only three Apollo lunar missions remaining, all possible care is being taken in the selection of the specific landing sites to insure the greatest scientific return. Figure 8 indicates the locations of some prime sites under consideration.

The Apollo 15 site is, of course, firm. The Lunar Module will land between the Hadley Rille and Apennine mountain front as shown on Figure 9. The broad objectives at this site are to study and sample the highland front, the adjacent mare and the rille itself. Figure 10 is a closer view of the site and the tentative traverses as they are now planned. The Apollo 14 astronauts walked a little more than $4 \mathrm{~km}$ on the surface. The traverses shown here total $37 \mathrm{~km}$. We feel the various improvements we have been able to make, in particular the roving vehicle, make feasible this excep- 


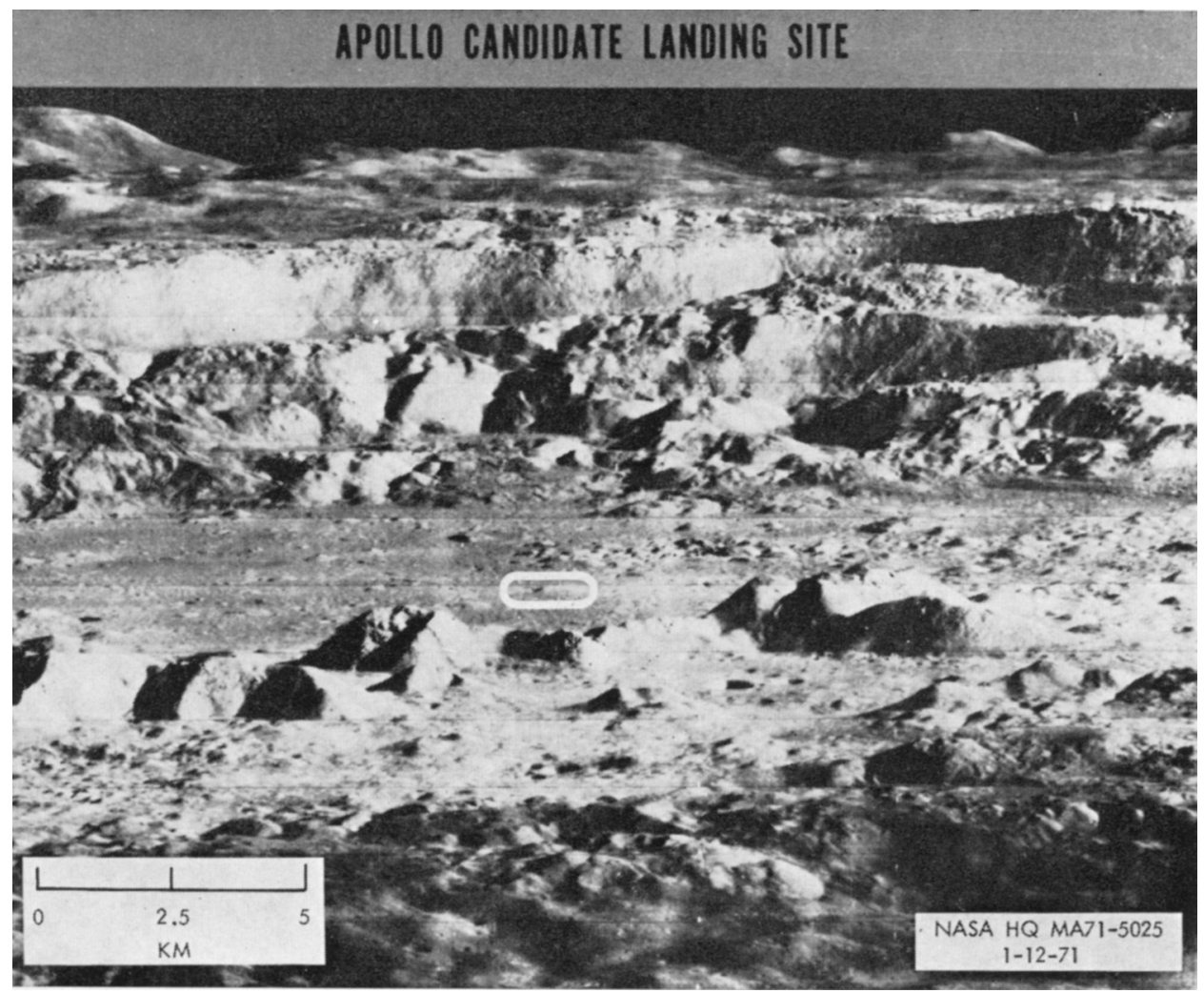

Fig. 12. Copernicus.

tional increase in exploration capability. As with each mission, a geophysical station will be left operating at this site. On the succeeding mission we plan to impact the spent booster out in Mare Imbrium. It is hoped that the resulting seismic data will be significant in unraveling the major mascon that exists in the Imbrium basin.

The last two landing sites have not as yet been selected. A great deal of photography was taken of highland regions around the Descartes site and to the eastward on Apollo 14 (Figure 11). These are now undergoing careful study and it is quite likely that Apollo 16 will be directed somewhere in this highland area. The Apollo 17 site will not be selected until about the end of 1971 .

Two of the sites under consideration are shown in Figures 12 and 13. At the spectacular crater Copernicus the landing site would be on the floor near the central peaks. Sampling these peaks would probably provide material from deep in the interior since they represent rebound from the initial impact. Marius Hills is an extensive volcanic region which lies along a major fault line of the moon. Even the Crater Tycho is not excluded from our considerations at this time although it obviously is a very difficult site operationally. 


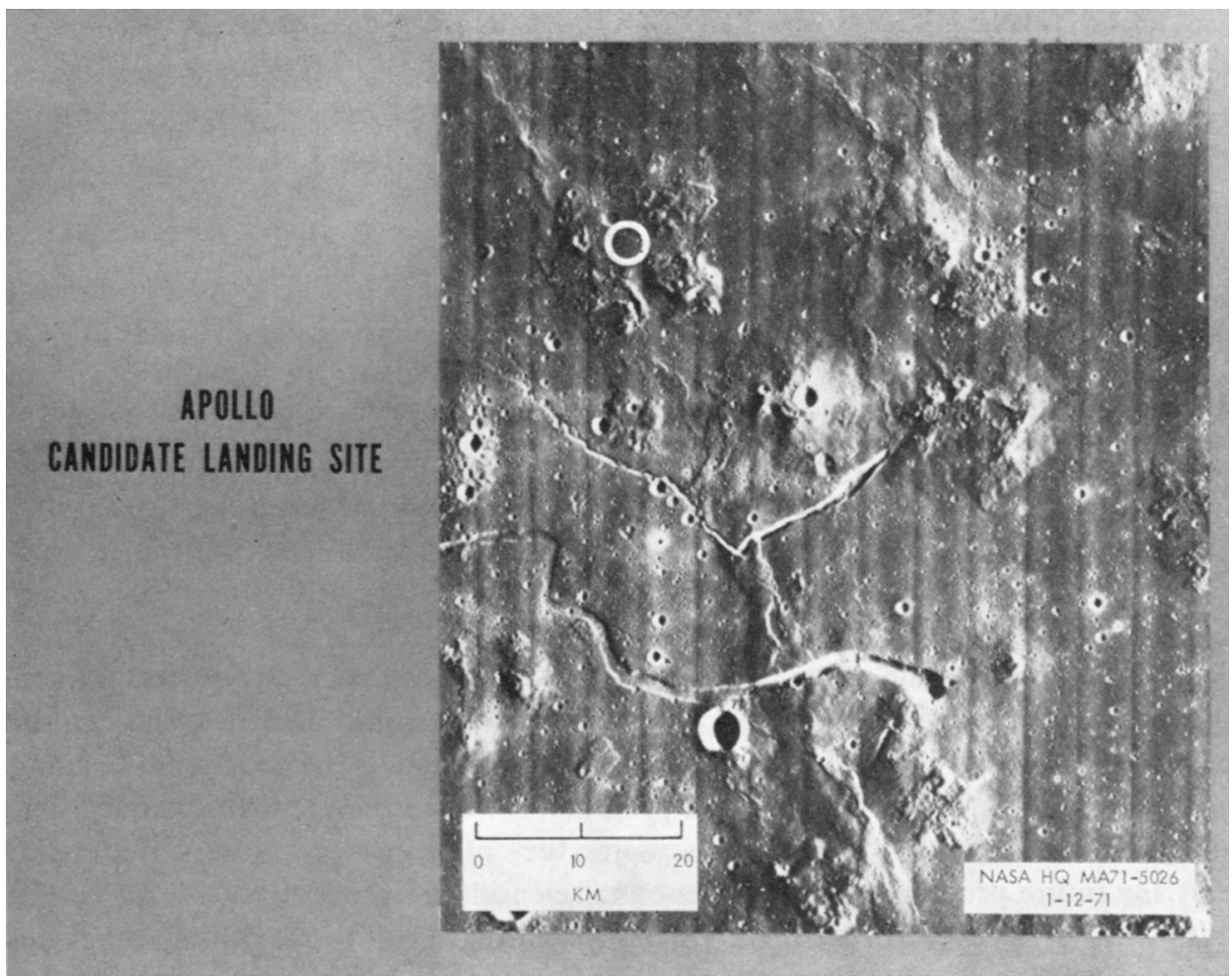

Fig. 13. Marius Hills.

In site selection, crew safety is always our primary consideration, of course. There are a number of other operational constraints that play a role; in particular, the availability of sufficient photography. Within the constraints we have, we want to take every feasible step to ensure the sites selected offer the highest potential for scientific gain. Any suggestions from qualified groups or individuals such as those present are most welcome. 